double-blind, phase 3 ENLIGHTEN-2 study comparing weight gain with OLZ/SAM vs olanzapine were eligible for ENLIGHTEN-2-EXT enrollment. Initial OLZ/SAM doses were based on olanzapine dose (10 or $20 \mathrm{mg}$ ) received at the conclusion of ENLIGHTEN-2; subsequent olanzapine dose adjustments were allowed. The samidorphan dose $(10 \mathrm{mg})$ remained fixed throughout. Assessments included adverse events (AEs), weight, waist circumference, metabolic laboratory parameters, and Positive and Negative Syndrome Scale (PANSS) scores. Analyses were based on observed results using descriptive statistics. Baseline was relative to the first OLZ/SAM dose in the extension study.

Results. 265 patients received OLZ/SAM; 167 (63.0\%) completed the extension study. Common AEs $(=5 \%)$ were weight decreased $(n=23 ; 8.7 \%)$, extra dose administered $(n=21 ; 7.9 \%)$, headache $(\mathrm{n}=18 ; 6.8 \%)$, and weight increased $(\mathrm{n}=16 ; 6.0 \%)$. At week 52 , mean (SD) change from baseline for weight and waist circumference was $-0.03(6.216) \mathrm{kg}$ and $-0.35(6.115) \mathrm{cm}$, respectively. Changes in fasting lipid and glycemic parameters were generally small and remained stable over 52 weeks. PANSS total scores remained stable during the extension.

Conclusions. OLZ/SAM was generally well tolerated over 52 weeks. Weight, waist circumference, metabolic laboratory parameters, and schizophrenia symptoms remained stable throughout the study.

Funding. Alkermes, Inc.

\section{Deutetrabenazine Reduces Severe Tardive Dyskinesia Movements in a 3-year Open- Label Extension Trial}

\author{
Nayla Chaijale, $\mathrm{PhD}^{1}$, Joseph Bona, $\mathrm{MD}^{2 \dagger}$, \\ Hadas Barkay, $\mathrm{PhD}^{3}$, Amanda Wilhelm, $\mathrm{PhD}^{1}$ and \\ Mark Forrest Gordon, MD ${ }^{1}$
}

${ }^{1}$ Teva Pharmaceutical Industries Ltd., West Chester, PA, USA, ${ }^{2}$ Emory University, Atlanta, GA, USA, and ${ }^{3}$ Teva Pharmaceutical Industries Ltd., Netanya, Israel

Presenting Author: Nayla Chaijale
Results. 337 patients were analyzed. The upper quartile of baseline total AIMS score was 14. Subgroups were defined as $>14$ and $\leq 14$ at baseline, respectively ( $\mathrm{n}=64$ vs 273 ); data are presented at Week $145(n=40$ vs 120$)$. Mean treatment duration was 880.5 and 760.8 days. Mean \pm SE daily doses were $41.1 \pm 1.6 \mathrm{mg}$ and $38.9 \pm 1.0 \mathrm{mg}$. Mean $\pm \mathrm{SE}$ change from baseline in AIMS score was $-11.0 \pm 0.8$ versus $-5.1 \pm 0.3$; percent change from baseline was $-60.1 \% \pm 3.6 \%$ versus $-55.9 \% \pm 3.0 \%$. More patients with AIMS score $>14$ had $\geq 50 \%$ AIMS reduction ( $73 \%$ vs $65 \%$ ). Less patients discontinued ( $38 \%$ vs $51 \%$ ); reasons included withdrawal by subject ( $16 \%$ vs $25 \%$ ), adverse event ( $3 \%$ vs $11 \%$ ), and lost to follow-up (6\% vs $7 \%)$. Withdrawal due to lack of efficacy was uncommon ( $5 \%$ vs $2 \%)$.

Conclusions. Patients with baseline total AIMS score $>14$ had clinically meaningful reductions in AIMS score, suggesting deutetrabenazine has long-term benefit in these patients.

Funding. Teva Pharmaceutical Industries Ltd., Petach Tikva, Israel

${ }^{\dagger}$ Dr. Bona died on June 1, 2020. Prior to his passing, Dr. Bona was instrumental in designing the post-hoc analyses described in this abstract and interpreted the interim data results for these analyses; however, he was not able to finalize this abstract.

\section{Qualitative Clinical Trial Exit Interviews Evaluating Treatment Benefit, Burden, and Satisfaction in Patients with Schizophrenia}

\author{
Adam Simmons, $\mathrm{MPH}^{1}$, Julia Carpenter-Conlin, $\mathrm{MSW}^{1}$, \\ Leona Bessonova, $\mathrm{PhD}^{1}$, Amy K. O'Sullivan, $\mathrm{PhD}^{1}$, \\ David McDonnell, $\mathrm{MD}^{2}$, Cory Saucier, $\mathrm{MPH}^{3}$, \\ Michelle K. White, $\mathrm{PhD}^{3}$, April M. Foster, $\mathrm{BS}^{3}$, \\ Jakob B. Bjorner, MD, $\mathrm{PhD}^{3}$, Olga Lapeyra, MD, CCRP ${ }^{4}$ and \\ David P. Walling, PhD $^{5}$
}

\footnotetext{
${ }^{1}$ Alkermes, Inc., Waltham, MA, USA, ${ }^{2}$ Alkermes Pharma Ireland Limited, Dublin, Ireland, ${ }^{3}$ Optum, Inc., Johnston, RI, USA, ${ }^{4}$ Segal Trials, Miami, FL, USA, and ${ }^{5} \mathrm{CNS}$ Network, LLC, Garden Grove, CA, USA
}

Presenting Author: Adam Simmons

\begin{abstract}
Background. There are no established treatment guidelines for tardive dyskinesia (TD) based on movement severity. The 12-week ARM-TD and AIM-TD studies in TD patients with baseline Abnormal Involuntary Movement Scale (AIMS) total score (items $1-7$ ) $\geq 6$ showed clinically significant improvements in AIMS score with deutetrabenazine versus placebo. Patients who completed these studies were eligible for the open-label extension (OLE) trial. This post-hoc analysis evaluated deutetrabenazine in TD patients with severe movements.

Methods. Subgroups were defined by upper quartile of baseline total AIMS score (local rating). Endpoints were: change and percent change from baseline in AIMS score, and percent of patients achieving $\geq 50 \%$ AIMS reduction from baseline.
\end{abstract}


Results. All 41 patients reported a lifetime burden with schizophrenia adversely impacting employment, relationships, emotional health, social activities, and daily tasks. Hospitalization for schizophrenia management was another reported aspect of disease burden. Although most $(n=32)$ patients reported previous medication benefits, side effects affecting physical, emotional/behavioral, and cognitive functioning were reported by all $(n=41)$. Following OLZ/SAM treatment, 39/41 patients (95\%) reported improvements in symptoms including hallucinations, paranoia, depression, sleep, and concentration. Furthermore, patients described improvements in self-esteem, social activities, relationships, and daily activities. Twenty-three patients (56\%) reported side effects attributed to OLZ/SAM; lack of energy $(\mathrm{n}=12[29 \%])$ and dry mouth $(\mathrm{n}=5$ [12\%]) were most common. Twenty-four (59\%) patients were "very satisfied" with OLZ/SAM; most $(\mathrm{n}=35[85 \%])$ preferred to continue OLZ/SAM vs switching to another medication. As most substudy patients $(\mathrm{n}=40 ; 98 \%)$ completed the extension study, satisfied patients may be overrepresented in this analysis.

Conclusion. This qualitative interview approach provided valuable insight into patients' experiences with previous medications and OLZ/SAM. Overall, most patients reported treatment satisfaction and improvements in symptoms, function, and healthrelated quality of life with OLZ/SAM.

Funding. Alkermes, Inc. by demographic characteristics. Case-control comorbidity comparisons were performed using prevalence rate ratios (PRRs) and 95\% CIs. Per-database medication exposure ( $=1$ National Drug Code in outpatients grouped by Redbook classification) was also assessed.

Results. Schizophrenia prevalence was $0.11 \%$ and $0.99 \%$ in commercially and Medicaid-insured patients, respectively. In both databases, comorbidity prevalence was higher among schizophrenia cases versus controls in approximately $=80 \%$ of the CCS level 2 categories assessed. Common top categories of comorbidities for schizophrenia cases were mood disorders, anxiety disorders, other connective tissue disease, and diseases of the heart. Comorbidities with the highest case-control PRRs included personality disorders, suicide and intentional self-inflicted injury, and impulse control disorders. Across databases, the most commonly prescribed medications in cases were antipsychotics, antidepressants, and analgesics/antipyretics opiate agonists; the most highly prescribed antipsychotics were risperidone, quetiapine, aripiprazole, and olanzapine.

Conclusions. This large-scale analysis quantifies the high prevalence of medical and psychiatric comorbidity burden in patients with schizophrenia, highlighting the importance of integrated medical and psychiatric care.

Funding. Alkermes, Inc.

\section{Disease Prevalence, Comorbid Conditions, and Medication Utilization Among Patients with Schizophrenia in the United States}

\section{Brittany Roy, $\mathrm{MPH}^{1}$, Ankitaben Shah ${ }^{1}$, Gary Bloomgren, $\mathrm{MD}^{1}$, Made Wenten, $\mathrm{PhD}, \mathrm{MPH}^{1}$, Jianheng Li, $\mathrm{MPH}^{2}$ and Cathy Lally, $\mathrm{MSPH}^{2}$}

${ }^{1}$ Alkermes, Inc., Waltham, MA, USA, and ${ }^{2}$ Epidemiologic Research \& Methods, LLC,

Presenting Author: Brittany Roy Atlanta, GA, USA

\section{Long-Term Safety and Efficacy of Deutetrabenazine in Younger and Older Patients With Tardive Dyskinesia}

Martha Sajatovic, $\mathrm{MD}^{1}$, Amanda Wilhelm, $\mathrm{PhD}^{2}$, Stacy Finkbeiner, $\mathrm{PhD}^{2}$, Hadas Barkay, $\mathrm{PhD}^{3}$, Nayla Chaijale, $\mathrm{PhD}^{2}$, Nicholas Gross, $\mathrm{MS}^{2}$ and Mark Forrest Gordon, MD ${ }^{2}$

${ }^{1}$ University Hospitals Cleveland Medical Center, Case Western Reserve University School of Medicine, Cleveland, OH, USA, ${ }^{2}$ Teva Pharmaceutical Industries Ltd., West Chester, PA, USA, and ${ }^{3}$ Teva Pharmaceutical Industries Ltd., Netanya, Israel

Presenting Author: Stacy Finkbeiner

\begin{abstract}
Objective. Disease prevalence, comorbid conditions, and pharmacological treatments were examined in a large population of US commercial- or Medicaid-insured individuals with schizophrenia.

Methods. This retrospective, cross-sectional claims analysis sourced data from the IBM MarketScan Commercial and Medicare Supplemental Databases and the Multi-state Medicaid Database (01Jan2009 to 30Jun2016). Cases were defined by $=1$ diagnostic claim (ICD-9-CM/ICD-10-CM) for schizophrenia during the study period. Comorbidities (=1 ICD-9-CM/ICD-10-CM diagnosis code) were grouped according to Clinical Classifications Software (CCS) level 2 categories. For the per-database analysis of comorbidities, schizophrenia cases were matched with controls
\end{abstract}

\begin{abstract}
Background. Tardive dyskinesia (TD) is an involuntary movement disorder that is more prevalent in older patients. However, there is limited information on TD treatment for this population. In two 12-week pivotal trials (ARM-TD and AIM-TD), TD patients demonstrated significant improvements in Abnormal Involuntary Movement Scale (AIMS) score with deutetrabenazine versus placebo.

Methods. Patients who completed ARM-TD or AIM-TD enrolled in an open-label extension (OLE) study. This post hoc analysis assessed change and percent change from baseline in AIMS score, response rates for $\geq 50 \%$ AIMS improvement, Patient Global Impression of Change (PGIC), Clinical Global
\end{abstract}

